\title{
Association of novel polymorphisms in TMEM39A gene with systemic lupus erythematosus in a Chinese Han population
}

\author{
Xinze Cai ${ }^{1}$, Wenyue Huang ${ }^{1}$, Xudong Liu', Lining Wang ${ }^{2}$ and Yi Jiang ${ }^{3 *}$
}

\begin{abstract}
Background: This study aimed to assess the association between 14 single nucleotide polymorphisms (SNPs) in six genes (IRF8, TMEM39A, IKZF3, ORMDL3, GSDMB, and ZPBP2) and systemic lupus erythematosus (SLE) in a Chinese Han population sample.

Methods: We carried out a case-control study of 415 patients with SLE and 470 healthy controls without autoimmune disease or cancer. DNA for genetic analysis was isolated from the blood of all subjects using standard phenol-chloroform method. TagSNPs were identified using genotype data from the panel (Han Chinese in Beijing) of the HapMap Project and were selected using the Haploview program. Genotyping assay was conducted using the Sequenom MassARRAY iPLEX Gold platform. The frequencies of the alleles and genotypes were calculated and analyzed. Association studies and haplotype analysis were also performed.

Results: The genotypic frequencies of rs12493175 and rs13062955 were significantly different between the SLE patients and the healthy controls. Compared with the common homozygous genotype, the $C T$ and $C T+\Pi$ genotypes in rs12493175 and the AC and AC + AA genotypes in rs13062955 was observed to significantly reduce the risk of SLE. The haplotype analysis of TMEM39A polymorphisms showed that the CGTA haplotype frequency was significantly low in the SLE patients.

Conclusion: Our findings identified three novel associations in SNPs located in the TMEM39A gene associated with SLE susceptibility in a Chinese Han population.
\end{abstract}

Keywords: Single nucleotide polymorphism, Systemic lupus erythematosus, Susceptibility

\section{Background}

Systemic lupus erythematosus (SLE) is typically characterized by the dysregulation of $\mathrm{T}$ cell response and $\mathrm{B}$ cell activation which usually causes the formation of immune complexes in multiple organs and tissues [1]. Although the pathogenesis of SLE is largely unknown to date, it most likely involves environmental and genetic factors. Several candidate-gene studies and genome-wide association (GWA) scans have successfully discovered multiple susceptibility genes that fall into key pathways implicating immune complex clearance, immune signal transduction and interferon pathways contributing to the

\footnotetext{
* Correspondence: xzcai@mail.cmu.edu.cn

${ }^{3}$ Central Laboratory; Department of Dermatology, First Affiliated Hospital of China Medical University, Nanjingbei Street, Heping District, Shenyang 110001, China

Full list of author information is available at the end of the article
}

development of SLE [2, 3]. However, much of the heritable risk needs to be identified.

Recently, multiethnic approach was utilized to find that three SLE risk loci exceeded the genome-wide significance threshold, including interferon regulatory factor 8 (IRF8), transmembrane protein 39A (TMEM39A), and $17 \mathrm{q} 21$ between IKAROS family of zinc finger 3 (IKZF3) and zona pellucida binding protein 2 (ZPBP2) [4]. The $17 \mathrm{q} 21$ region was originally associated with asthma in family linkage study [5-7]. More single nucleotide polymorphisms (SNPs) in the $17 \mathrm{q} 21$ region have been identified as being associated with the susceptibility to autoimmune diseases, including rheumatoid arthritis, ankylosing spondylitis and SLE [4, 8-10]. IRF8 is a family member of transcription factors that play a critical role in the regulation of cell apoptosis and 
immune response [11]. It is required for promoting type I interferon responses which can induce the overexpression of genes reported in SLE, and several variants within IRF8 could influence binding to the regulatory elements $[4,12]$. Although very limited biological data of TMEM $39 A$ is published so far, its polymorphisms have been found to be associated with multiple sclerosis and SLE [4, 13-15]. Additionally, several studies found genetic variants in orosomucoid like 3 (ORMDL3) and gasdermin B (GSDMB) were associated with the risk of autoimmune disease $[16,17]$. On the basis of these studies, we hypothesized that certain novel variants in the loci described previously may contribute to the susceptibility to SLE.

In this study, we selected the six candidate genes, namely, IRF8, TMEM39A, IKZF3, ORMDL3, GSDMB, and $Z P B P 2$, and screened for the putatively functional tagSNPs. We aimed to determine the association between the polymorphisms and susceptibility to SLE in a Chinese population.

\section{Methods}

\section{Sample description}

A total of 415 patients with SLE diagnosed according to the criteria of the 1982 American College of Rheumatology were enrolled [18]. Additionally, 470 healthy controls without autoimmune disease or cancer were recruited, who were sex- and age-matched with the patients. All the study participants were from the Chinese Han population, with the age ranging from 16 to 65 years. Demographic and clinical characteristics of SLE patients and controls are shown in Table 1. This project was approved by the Human Ethics Review Committee of China Medical University. Written informed consent was obtained from all the participants, including the guardians on behalf of the children enrolled in the study.

Table 1 General characteristics of the study population

\begin{tabular}{llll}
\hline & $\begin{array}{l}\text { SLE patients } \\
(n=415)\end{array}$ & $\begin{array}{l}\text { Healthy controls } \\
(n=470)\end{array}$ & $P$ value \\
\hline Female/male & $312(103)$ & $362(108)$ & $P>0.05$ \\
Age (mean \pm SD), (years) & $38.2 \pm 11.4$ & $35.8 \pm 13.6$ & $P>0.05$ \\
Fever, n (\%) & $57(13.7)$ & - & \\
Baldness, n (\%) & $148(35.7)$ & - & \\
Light sensitivity, n (\%) & $74(17.8)$ & - & \\
Facial erythema, n (\%) & $161(38.8)$ & - & \\
Oral ulcer, n (\%) & $67(16.1)$ & - & \\
Arthritis, n (\%) & $62(14.9)$ & - & \\
Lupus nephritis, n (\%) & $246(59.3)$ & - & \\
\hline
\end{tabular}

\section{SNP selection}

TagSNPs are representative SNPs which can capture most of the genetic variation in a region of the genome on the basis that they are in high linkage disequilibrium (LD) with other SNPs [19]. TagSNPs genotyped in this study were selected by analyzing the genotype data of Chinese Han population from HapMap dbSNP (http://www.hapmap.ncbi.nlm.nih.gov) using LD-based tagSNP selection with a pairwise algorithm LDSelect, available in the Tagger function implemented in Haploview version 4.2 (http://www.broadinstitute.org/ mpg/haploview) [20, 21].

First, genotype data of HapMap Chinese Han Beijing population (Release 27, Phase I + II + III) were extracted and the chromosomal regions including the six candidate genes within the extended gene regions encompassing $3000 \mathrm{bp}$ upstream and $1500 \mathrm{bp}$ downstream flanking sequence (to capture the 5' and 3' UTR) were searched. TagSNPs were chosen based on a minor allele frequency (MAF) of at least $5 \%$ and a pairwise LD threshold of $r^{2}>0.8$ using Haploview 4.2. Second, the F-SNP program (http://compbio.cs.queensu.ca/F-SNP) and SNP Function Prediction (FuncPred) software (http://snpinfo.niehs. nih.gov/snpinfo/snpfunc.htm) were applied to prioritize the tagSNPs for genotyping based on their putative functions. Accordingly, 14 tagSNPs with predicted functional effects were selected for genotyping. The common SNPs captured using the selected tagSNPs in the six candidate genes are presented in Table 2.

\section{Genotyping assay}

Genomic DNA was isolated from peripheral blood leukocytes using the standard phenol-chloroform method. Each DNA sample was diluted to working concentration of $50 \mathrm{ng} / \mu \mathrm{l}$ for genotyping. The selected tagSNP genotyping was performed by BGI (Shenzhen, China) using the Sequenom MassARRAY iPLEX Gold platform (Sequenom, San Diego, California) according to the manufacturer's instructions [22]. The primers for polymorphism genotyping were designed using MassARRAY Assay Design 3.1 software and are shown in Table 3. All samples were randomized on 384-well plates and blinded for case or control status. A random selection of samples was repeatedly genotyped using direct sequencing validate the accuracy of the SNP genotyping assays and the results were $100 \%$ concordant.

Reverse transcriptase-PCR of candidate gene mRNA levels To examine the relation between the associated polymorphisms and the gene mRNA levels, forty patients stratified by polymorphic genotypes were randomly selected. The relative expression levels of twenty patients with common homogenous genotype carriers were set to a unity, and the relative expression levels of twenty 
Table 2 Common SNPs captured using the selected 14 tagSNPs in the six candidate genes based on the HapMap population data for Chinese in Beijing (Release 27)

\begin{tabular}{|c|c|c|c|}
\hline Gene & tagSNP_ID & SNP captured & Position (hg19) \\
\hline \multirow[t]{2}{*}{ IKZF3 } & rs3816470 & rs9635726, rs3816470, rs9303277, rs10445308, rs9909593 & chr17:37985801 \\
\hline & rs907092 & rs907092 & chr17:37922259 \\
\hline GSDMB & rs9303281 & $\begin{array}{l}\text { rs } 11078927, \text { rs } 1008723, \text { rs } 4795400, \text { rs } 9303281, \text { rs2305480, rs7219923, } \\
\text { rs } 869402, \text { rs2305479, rs7224129, rs } 11078926, \text { rs2290400, rs7216389 }\end{array}$ & chr17:38074046 \\
\hline \multirow[t]{2}{*}{ ORMDL3 } & rs4795402 & rs4795403, rs4795402, rs3744246, rs4795404 & chr17:38085385 \\
\hline & rs8076131 & rs4378650, rs8076131, rs12603332 & chr17:38080912 \\
\hline ZPBP2 & rs11557466 & $\begin{array}{l}\text { rs11557467, rs12936231, rs11078925, rs1054609, rs11557466, } \\
\text { rs11870965, rs10852936, rs9907088 }\end{array}$ & chr17:38024626 \\
\hline \multirow[t]{4}{*}{ IRF8 } & rs188602 & rs188602, rs170033, rs2270502, rs381139 & chr16:85932351 \\
\hline & rs4843860 & rs4843860, rs12926854, rs4843861 & chr16:85950921 \\
\hline & rs2270501 & rs2270501, rs12924316 & chr16:85932988 \\
\hline & rs191022 & rs191022 & chr16:85932132 \\
\hline \multirow[t]{3}{*}{ TMEM39A } & rs13062955, rs12493175 & $\begin{array}{l}\text { rs 12492859, rs13094625, rs13081197, rs13078312, rs12493326, } \\
\text { rs16829853, rs13081067, rs2282170, rs13062955, rs12492315, } \\
\text { rs12493175, rs13096213, rs12496277, rs12492609 }\end{array}$ & chr3:119159658,chr3:119160413 \\
\hline & rs4687859 & $\begin{array}{l}\text { rs7629750, rs2282171, rs3772136, rs9846088, rs4687859, } \\
\text { rs9872589, rs3195852 }\end{array}$ & chr3:119170371 \\
\hline & rs2282175 & rs17281647, rs2282175, rs1132202 & chr3:119182259 \\
\hline
\end{tabular}

patients with heterogeneous and rare homogenous genotypes were expressed relative to those of the common homogenous genotype carriers. Total RNA prepared from peripheral blood mononuclear cells were reversely transcribed using the TaqMan reverse transcription reagents (Applied Biosystems, Weiterstadt, Germany). RTPCR was carried out using the ABI Universal Master Mix on an ABI PRISM 7000 Sequence Detection System.

\section{Statistical analysis}

Data were managed and stored using the SPSS software 16.0. Allele and genotype frequencies were compared between patient and control groups by the chi-square $\left(\chi^{2}\right)$ test. The quality of the genotype data was evaluated by Hardy-Weinberg equilibrium (HWE) in the case and control subjects using Fisher's exact test $(P>0.05)$. The association between each polymorphism and risk of SLE was estimated by logistic regression and was expressed as odds ratio (OR) with 95\% confidence intervals (95\% $\mathrm{CI})$. The haplotypes were assigned using the online software platform SHEsis (http://www.analysis.bio-x.cn). The haplotype construction element is based on the standard Full-Precise-Iteration (FPI) algorithm [23]. All tests were two-tailed, and $P$ values $<0.05$ were considered as statistically significant.

\section{Results}

In the screening stage, a few of tagSNPs for the six candidate genes were excluded from further analysis because they were found to have no polymorphic sites or to exhibited MAFs $<0.05$ in Chinese Han Beijing

Table 3 Details of the primers used in the polymorphism genotyping by MassArray

\begin{tabular}{|c|c|c|c|}
\hline tagSNP_ID & Alleles & Forward and reverse primer & Extension primer \\
\hline \multirow[t]{2}{*}{ rs2282175 } & $\mathrm{C} / \mathrm{T}$ & ACGTTGGATGGAAAGCGGCGACAACTTTAC & CGCTGGGAGGGAGTTC \\
\hline & & ACGTTGGATGCTGGTTTGCAGCGTTCCAAC & \\
\hline \multirow[t]{2}{*}{ rs4687859 } & $A / G$ & ACGTTGGATGCATGCCTGGCCTCATTITC & TTTCCCTGCCTCATTG \\
\hline & & ACGTTGGATGAGAAAGCACATTTCCCTGCC & \\
\hline \multirow[t]{2}{*}{ rs12493175 } & $C / T$ & ACGTTGGATGGTTATGGGACAGCTTCTTTC & CCCAAACGTATGAAGGTTAACAG \\
\hline & & ACGTTGGATGGAGAGGTGAGAAAGCTACAG & \\
\hline \multirow[t]{2}{*}{ rs13062955 } & $\mathrm{A} / \mathrm{C}$ & ACGTTGGATGGGCAAATACAGGCATACCTC & GGACTACAGTATCTGGGAAGCACAAT \\
\hline & & ACGTTGGATGGGGTTGCCACAAACCTTCAG & \\
\hline \multirow[t]{2}{*}{ rs9303281 } & $A / G$ & ACGTTGGATGACCCCTTITTTGGACTCAGC & СTCTTCCATGTGAAGAGAGTCCA \\
\hline & & ACGTTGGATGACGTGCGTCCATGTGAAGAG & \\
\hline
\end{tabular}


population. Finally, 14 tagSNPs with predicted functional effects were selected for genotyping in a total of 885 subjects (Table 2). The details of the five identified genetic single-nucleotide variants in two genes, namely, TMEM39A rs2282175, rs4687859, rs12493175, rs13062955, and GSDMB rs9303281 were presented (Table 4). Additionally, there was no significant difference concerning the call rates between the SLE group and the control $(p>0.05)$. However, as shown in Table 5 , the allelic distributions of rs4687859 and rs9303281 showed significant departure from the Hardy-Weinberg law for the controls. The allelic distributions of the three selected tagSNPs, rs2282175, rs12493175, and rs13062955, of the TMEM39A gene met the Hardy-Weinberg principle (Table 5). Thus, we focused on the three selected tagSNPs, rs2282175, rs12493175, and rs13062955, of the TMEM39A gene in the following analysis (Table 6).

The genotypic frequencies of rs12493175 and rs13062955 located in TMEM39A gene were significantly different between the SLE patients and the healthy controls. Compared with the common homozygous genotype, the $\mathrm{CT}$ and $\mathrm{CT}+\mathrm{TT}$ genotypes in rs 12493175 (p.adjust $=0.005$, odds ratio (OR) 0.58, 95\% CI 0.42 to 0.79 ; p.adjust $=0.007$, OR $0.62,95 \%$ CI 0.46 to 0.84 , respectively) and the $\mathrm{AC}$ and $\mathrm{AC}+\mathrm{AA}$ genotypes in rs13062955 (p.adjust $=0.002$, OR $0.55,95 \%$ CI 0.40 to 0.76 ; p.adjust $=0.007$, OR $0.61,95 \%$ CI 0.45 to 0.84 , respectively) was observed to significantly reduce the risk of SLE. On the other hand, the difference in the frequency of rs2282175 was only marginal. The $\mathrm{CT}$ and CT + TT genotypes in rs2282175 was observed to modestly increase the risk of SLE (p.adjust $=0.054$, OR $1.63,95 \%$ CI 1.06 to 2.38; p.adjust $=$ 0.054 , OR $1.56,95 \%$ CI 1.05 to 2.27 , respectively). However, we did not find any other tagSNP associated with SLE risk in the genes of IRF8, IKZF3, $O R M D L 3, G S D M B$ and $Z P B P 2$. We also evaluated the relation between the associated polymorphisms and the gene mRNA levels in peripheral blood mononuclear cells from 40 patients. Nevertheless, we failed to find any correlation between them (data not shown).

Table 4 The details of the identified genetic single-nucleotide variants

\begin{tabular}{lllll}
\hline SNP & Chr & Position & Func.refGene & CADD.Score \\
\hline rs13062955 & chr3 & 119159658 & intronic & CADD $=3.564$ \\
rs12493175 & chr3 & 119160413 & intronic & CADD $=2.429$ \\
rs4687859 & chr3 & 119170371 & intronic & CADD $=8.630$ \\
rs2282175 & chr3 & 119182259 & UTR5 & CADD $=4.988$ \\
rs9303281 & chr17 & 38074046 & intronic & CADD $=0.720$ \\
\hline
\end{tabular}

Func.refGene functional gene element, $C A D D$ Combined Annotation Dependent Depletion
Table 5 The five SNPs call rates in patients and control individuals and HWE $p$-values

\begin{tabular}{llllll}
\hline SNP_ID & \multicolumn{2}{l}{ Call rate (\%) } & & HWE $p$-value & \\
\cline { 2 - 3 } & SLE & Control & & SLE & Control \\
\hline rs2282175 & 98 & 99 & & 0.539349 & 0.056740 \\
rs4687859 & 98 & 95 & & 0.792124 & 0.000719 \\
rs12493175 & 99 & 99 & & 0.004372 & 0.295414 \\
rs13062955 & 97 & 92 & & 0.002761 & 0.107666 \\
rs9303281 & 93 & 99 & & $7.77 E-16$ & $2.66 E-15$ \\
\hline
\end{tabular}

Haplotypes were constructed in both SLE and healthy controls and the haplotypes with frequency of $>3 \%$ were built from TMEM39A rs2282175, rs12493175 and rs13062955 (Table 7). The results show that the CGTA haplotype frequency was significantly low in the SLE patients $(p=0.019$, OR $0.72,95 \%$ CI 0.55 to 0.95$)$. No difference was detected in the other haplotypes.

\section{Discussion}

IRF8, TMEM39A and IKZF3-ZPBP2 were previously identified as susceptibility loci for SLE in the multiracial replication study [4], Besides, ORMDL3 and GSDMB were found to have susceptibility loci for autoimmune diseases $[16,17]$. Thus we hypothesized certain novel associations in SNPs located in these genes could be identified in Chinese populations. To test this hypothesis, we selected 14 tagSNPs in these candidate genes to determine the association between the polymorphisms and SLE susceptibility in a Chinese Han population. Our findings showed that TMEM39A rs2282175, rs12493175, and rs13062955 were associated with SLE risk.

To date, almost no biological data of TMEM39A have been reported and only two SNPs in TMEM39A were identified as being associated with the susceptibility of autoimmune diseases. TMEM39A rs1132200 have been found to be associated with susceptibilities to multiple sclerosis and SLE in multiracial replication study $[4,13,14]$. but the recent studies showed that TMEM39A rs12494314, instead of rs1132200, was associated with SLE susceptibility in the Chinese population [15, 24]. In our current study, we identified three novel associations in SNPs located in TMEM39A as being associated with SLE susceptibility. The genotypic frequencies of rs12493175 and rs13062955 were significantly different between the SLE patients and the healthy controls, while the difference in the frequency of rs2282175 was only marginal. Among these polymorphisms, rs12493175 T-allele and rs13062955 Aallele were found to be associated with a reduced SLE risk, suggesting a protective factor to SLE. In contrast, rs2282175 T-allele was found to be associated with an increased SLE risk, suggesting a susceptibility factor to SLE. Haplotype analysis for TMEM39A SNPs revealed that the haplotype CGTA conferred a reduced risk of SLE. It is 
Table 6 Genotype and allele association analysis of three tagSNPs

\begin{tabular}{|c|c|c|c|c|c|c|c|c|}
\hline tagSNP_ID & Genotype/Allele & SLE, n(\%) & $\mathrm{CON}, \mathrm{n}(\%)$ & $x^{2}$ & $P$ value & OR (95\% Cl) & $P$ value & P.adjust \\
\hline \multirow[t]{6}{*}{ rs2282175 } & CC & $339(83.7)$ & $415(88.9)$ & 5.1 & 0.08 & 1 & & \\
\hline & $\mathrm{CT}$ & $62(15.3)$ & $48(10.3)$ & & & $1.63(1.06-2.38)$ & 0.026 & 0.054 \\
\hline & $\pi$ & $4(1.0)$ & $4(0.9)$ & & & $1.21(0.30-5.00)$ & 0.776 & 0.817 \\
\hline & $\mathrm{CT} / \mathrm{TT}$ & & & & & $1.56(1.05-2.27)$ & 0.027 & 0.054 \\
\hline & $C$ & 740 (91.4) & $878(94.0)$ & 4.5 & 0.033 & 1 & & \\
\hline & $\mathrm{T}$ & $70(8.6)$ & $56(6.0)$ & & & $1.49(1.03-2.12)$ & 0.033 & 0.06 \\
\hline \multirow[t]{6}{*}{ rs12493175 } & $\mathrm{CC}$ & $311(75.7)$ & 308 (66.2) & 12.7 & 0.002 & 1 & & \\
\hline & $\mathrm{CT}$ & $85(20.7)$ & $145(31.2)$ & & & $0.58(0.42-0.79)$ & 0.001 & 0.005 \\
\hline & $\pi$ & $15(3.6)$ & $12(2.6)$ & & & $1.23(0.57-2.70)$ & 0.589 & 0.736 \\
\hline & $\mathrm{CT} / \mathrm{TT}$ & & & & & $0.62(0.46-0.84)$ & 0.002 & 0.007 \\
\hline & C & 707 (86.0) & 761 (81.8) & 5.6 & 0.017 & 1 & & \\
\hline & $\mathrm{T}$ & $115(14.0)$ & 169 (18.2) & & & $0.73(0.56-0.95)$ & 0.017 & 0.0486 \\
\hline \multirow[t]{6}{*}{ rs13062955 } & CC & 305 (75.9) & $281(66.0)$ & 15.1 & 0.001 & 1 & & \\
\hline & $A C$ & $82(20.4)$ & $136(31.9)$ & & & $0.55(0.40-0.76)$ & $2.95 \times 10^{-4}$ & 0.002 \\
\hline & AA & $15(3.7)$ & $9(2.1)$ & & & $1.53(0.66-3.57)$ & 0.318 & 0.424 \\
\hline & $\mathrm{AC} / \mathrm{AA}$ & & & & & $0.61(0.45-0.84)$ & 0.002 & 0.007 \\
\hline & $C$ & $692(86.1)$ & 698 (81.9) & 5.2 & 0.021 & 1 & & \\
\hline & $A$ & $112(13.9)$ & $154(18.1)$ & & & $0.73(0.56-0.96)$ & 0.021 & 0.053 \\
\hline
\end{tabular}

P.adjust: the Bonferroni corrected $P$ value

possible that the haplotype CGTA provides protection to SLE, resulting from the rs12493175 T and rs13062955 A alleles. It is worth noting that rs2282175 is located in the region of $5^{\prime}$ upstream in TMEM39A and predicted to be a binding site of certain transcription factor. It is speculated that the $\mathrm{C} \rightarrow \mathrm{T}$ allele change at the rs2282175 site may influence the DNA binding ability of transcription factor cRel, which was predicted according to the different variants using the search tool of Alibaba 2.1 (http:// www.gene-regulation.com/pub/programs/alibaba2). Although we did not find any relation between mRNA expression and the polymorphisms, it may be required to explore the possible biological significance of the SNPs in different cell subsets.

Several limitations in the current study should also be noted. First, due to the restricted number of study subjects and limited analysis capacity, we did not analyze the SNPs with rare MAF in the Chinese Han population, including those reported as risk loci for SLE in other ancestries, and mainly focused on the SNPs with predicted

Table 7 Frequencies of the haplotypes formed by TMEM39A rs2282175, rs12493175 and rs13062955 SNPs

\begin{tabular}{lllll}
\hline Haplotype & SLE, $\mathrm{n}(\%)$ & CON, $\mathrm{n}(\%)$ & $P$ value & OR $(95 \% \mathrm{Cl})$ \\
\hline CACC & $492.8(62.4)$ & $527.7(63.0)$ & 0.813 & $0.97(0.79-1.19)$ \\
CGCC & $119.4(15.1)$ & $103.3(12.3)$ & 0.101 & $1.26(0.95-1.68)$ \\
CGTA & $108.7(13.8)$ & $151.0(18.0)$ & 0.019 & $0.72(0.55-0.95)$ \\
TGCC & $65.5(8.3)$ & $52.7(6.3)$ & 0.11 & $1.34(0.92-1.96)$ \\
\hline
\end{tabular}

functional effect. Further research on the role of the rare variants should be carried out in a larger number of samples. As different populations have different genetic backgrounds, it is still necessary to perform the genetic analysis of multiracial study. Second, we still could not determine the causality of SLE-associated SNPs. For those variants in the large strong LD region, such as chromosome $17 \mathrm{q} 21$, it is difficult to determine which SNP is the true functional locus that contributes to SLE susceptibility independently. Better understanding whether the SNP is functionally relevant will require mechanistic and fine-mapping experiments. Third, our study did not assess the SNP-SNP interaction (epistasis). For the genetically complex disease, multiple interacting loci could contribute to SLE susceptibility. Additionally, as a heterogenetic disease, the contribution of genetic and environmental factors is very important for the disease [25]. Therefore, interaction between genetic and environmental factors is required to further clarify the pathogenesis of SLE.

\section{Conclusion}

This study identified three novel associations in SNPs located in the TMEM39A gene associated with SLE susceptibility in a Chinese Han population. Functional study and further independent large-scale study in other racial populations are still needed to confirm our results. 


\section{Abbreviations}

Cl: Confidence interval; GSDMB: Gasdermin B; GWA: Genome-wide association; IKZF3: IKAROS family of zinc finger 3; IRF8: Interferon regulatory factor 8; MAF: Minor allele frequency; OR: Odds ratio; ORMDL3: Orosomucoid like 3; SLE: Systemic lupus erythematosus; SNP: Single nucleotide polymorphism; TMEM39A: Transmembrane protein 39A; ZPBP2: Zona pellucida binding protein 2

\section{Acknowledgment}

We especially thank all SLE patients who participated for making this study possible.

\section{Funding}

The work was supported by grants from the National Natural Science Foundation of China (81401330) and the National Science and Technology Pillar Program (2011BAl10B04).

\section{Availability of data and material}

All data generated or analysed during this study are included in this published article.

\section{Authors' contributions}

XC contributed to the collection, analysis of the clinical data, and manuscript preparation. $\mathrm{WH}$ and $\mathrm{XL}$ contributed to the analysis of the SNP results. LW and YJ coordinated the study and helped draft the manuscript. All authors read and approved the final manuscript.

\section{Competing interests}

The authors declare that they have no competing interests.

\section{Consent for publication}

Not applicable.

\section{Ethics approval and consent to participate}

The study was approved by the Human Ethics Committee of China Medical University and the informed consents were obtained from all donors.

\section{Publisher's Note}

Springer Nature remains neutral with regard to jurisdictional claims in published maps and institutional affiliations.

\section{Author details}

${ }^{1}$ Central Laboratory, First Affiliated Hospital of China Medical University, Nanjingbei Street, Heping District, Shenyang 110001, China. ${ }^{2}$ Department of Nephrology, First Affiliated Hospital of China Medical University, Nanjingbei Street, Heping District, Shenyang 110001, China. ${ }^{3}$ Central Laboratory; Department of Dermatology, First Affiliated Hospital of China Medical University, Nanjingbei Street, Heping District, Shenyang 110001, China.

\section{Received: 20 July 2016 Accepted: 13 April 2017}

\section{Published online: 20 April 2017}

\section{References}

1. Rother N, van der Vlag J. Disturbed T cell signaling and altered Th17 and regulatory $T$ cell subsets in the pathogenesis of systemic lupus erythematosus. Front Immunol. 2015;6:610

2. Moser KL, Kelly JA, Lessard CJ, Harley JB. Recent insights into the genetic basis of systemic lupus erythematosus. Genes Immun. 2009;10(5):373-9.

3. Deng Y, Tsao BP. Genetic susceptibility to systemic lupus erythematosus in the genomic era. Nat Rev Rheumatol. 2010;6(12):683-92.

4. Lessard CJ, Adrianto I, Ice JA, Wiley GB, Kelly JA, Glenn SB, Adler AJ, Li H, Rasmussen A, Williams AH, et al. Identification of IRF8, TMEM39A, and IKZF3-ZPBP2 as susceptibility loci for systemic lupus erythematosus in a large-scale multiracial replication study. Am J Hum Genet. 2012;90(4): 648-60

5. Dizier MH, Besse-Schmittler C, Guilloud-Bataille M, Annesi-Maesano Boussaha M, Bousquet J, Charpin D, Degioanni A, Gormand F, Grimfeld A, et al. Genome screen for asthma and related phenotypes in the French EGEA study. Am J Respir Crit Care Med. 2000;162(5):1812-8.

6. Marinho S, Custovic A, Marsden P, Smith JA, Simpson A, 17q12-21 variants are associated with asthma and interact with active smoking in an adult population from the United Kingdom. Ann Allergy Asthma Immunol. 2012; 108(6):402-11. e409.

7. Fang Q, Zhao H, Wang A, Gong Y, Liu Q. Association of genetic variants in chromosome 17q21 and adult-onset asthma in a Chinese Han population. BMC Med Genet. 2011;12:133.

8. Qiu R, Zhang H, Zhao H, Li J, Guo C, Gong Y, Liu Q. Genetic variants on $17 q 21$ are associated with ankylosing spondylitis susceptibility and severity in a Chinese Han population. Scand J Rheumatol. 2013;42(6):469-72.

9. Kurreeman FA, Stahl EA, Okada Y, Liao K, Diogo D, Raychaudhuri S, Freudenberg J, Kochi Y, Patsopoulos NA, Gupta N, et al. Use of a multiethnic approach to identify rheumatoid- arthritis-susceptibility loci, 1 p36 and 17q12. Am J Hum Genet. 2012;90(3):524-32.

10. Cai X, Qiao Y, Diao C, Xu X, Chen Y, Du S, Liu X, Liu N, Yu S, Chen D, et al. Association between polymorphisms of the IKZF3 gene and systemic lupus erythematosus in a Chinese Han population. PLoS One. 2014;9(10), e108661.

11. Tamura T, Yanai H, Savitsky D, Taniguchi T. The IRF family transcription factors in immunity and oncogenesis. Annu Rev Immunol. 2008;26:535-84.

12. Baechler EC, Batliwalla FM, Karypis G, Gaffney PM, Ortmann WA, Espe KJ, Shark KB, Grande WJ, Hughes KM, Kapur V, et al. Interferon-inducible gene expression signature in peripheral blood cells of patients with severe lupus. Proc Natl Acad Sci U S A. 2003;100(5):2610-5.

13. (IMSGC) IMSGC. Comprehensive follow-up of the first genome-wide association study of multiple sclerosis identifies KIF21B and TMEM39A as susceptibility loci. Hum Mol Genet. 2010;19(5):953-62.

14. Varade J, Comabella M, Ortiz MA, Arroyo R, Fernandez O, Pinto-Medel MJ, Fedetz M, Izquierdo G, Lucas M, Gomez CL, et al. Replication study of 10 genes showing evidence for association with multiple sclerosis: validation of TMEM39A, IL12B and CBLB [correction of CLBL] genes. Mult Scler. 2012; 18(7):959-65.

15. Sheng YJ, Xu JH, Wu YG, Zuo XB, Gao JP, Lin Y, Zhu ZW, Wen LL, Yang C, $L i u L$, et al. Association analyses confirm five susceptibility loci for systemic lupus erythematosus in the Han Chinese population. Arthritis Res Ther. 2015;17:85.

16. Verlaan DJ, Berlivet $S$, Hunninghake $G M$, Madore $A-M$, Larivière $M$, Moussette S, Grundberg E, Kwan T, Ouimet M, Ge B. Allele-specific chromatin remodeling in the ZPBP2/GSDMB/ORMDL3 locus associated with the risk of asthma and autoimmune disease. Am J Hum Genet. 2009:85(3): 377-93.

17. Li X, Ampleford EJ, Howard TD, Moore WC, Torgerson DG, Li H, Busse WW Castro M, Erzurum SC, Israel E. Genome-wide association studies of asthma indicate opposite immunopathogenesis direction from autoimmune diseases. J Allergy Clin Immunol. 2012;130(4):861-868. e867.

18. Yu C, Gershwin ME, Chang C. Diagnostic criteria for systemic lupus erythematosus: a critical review. J Autoimmun. 2014:48-49:10-3.

19. Kathiresan S, Gabriel SB, Yang Q, Lochner AL, Larson MG, Levy D, Tofler GH, Hirschhorn JN, O'Donnell CJ. Comprehensive survey of common genetic variation at the plasminogen activator inhibitor-1 locus and relations to circulating plasminogen activator inhibitor-1 levels. Circulation. 2005:112(12): 1728-35.

20. Barrett JC, Fry B, Maller J, Daly MJ. Haploview: analysis and visualization of LD and haplotype maps. Bioinformatics. 2005;21(2):263-5.

21. Carlson CS, Eberle MA, Rieder MJ, Yi Q, Kruglyak L, Nickerson DA. Selecting a maximally informative set of single-nucleotide polymorphisms for association analyses using linkage disequilibrium. Am J Hum Genet. 2004; 74(1):106-20.

22. Yi X, Li J, Yu S, Zhang A, Xu J, Yi J, Zou J, Nie X, Huang J, Wang J. A new PCR-based mass spectrometry system for high-risk HPV, part I: methods. Am J Clin Pathol. 2011;136(6):913-9.

23. Yong $Y$, Lin H. SHEsis, a powerful software platform for analyses of linkage disequilibrium, haplotype construction, and genetic association at polymorphism loci. Cell Res. 2005:15(2):97-8.

24. You Y, Zhai ZF, Chen FR, Chen W, Hao F. Autoimmune risk loci of IL12RB2, IKZF1, XKR6, TMEM39A and CSK in Chinese patients with systemic lupus erythematosus. Tissue Antigens. 2015;85(3):200-3.

25. Sparks JA, Costenbader KH. Genetics, environment, and gene-environment interactions in the development of systemic rheumatic diseases. Rheum Dis Clin North Am. 2014;40(4):637-57. 